\title{
Arbor
}

\section{El Ejército del Aire en la estructura militar de la OTAN}

\section{Francisco Gómez Carretero}

arbor CLXXI, 674 (Febrero 2002), 401-417 pp.

La entrada de España en la estructura militar de la Alianza, la incorporación de nuevos miembros a ésta, los recientes acontecimientos del 11 de septiembre y la aplicación del Articulo $V$ del Tratado en el conflicto de Afganistán, hacen que todo lo relacionado con esta Organización sea de plena actualidad.

El artículo trata dicha Organización de forma general, para centrarse en las Fuerzas Aliadas en Europa Meridional (AFSOUTH), en especial en las Fuerzas Aéreas Aliadas del Sur (AIRSOUTH) y sus relaciones con el Ejército del Aire. Se nos da una visión general de estas relaciones, profundizando en lo relativo al Centro Combinado de Operaciones Aéreas de Torrejón (CAOC 8), y en los ejercicios de Puestos de Mando (CPX) o reales (LIVEX), en los que participa el Ejército del Aire.

También trata sobre la puesta en práctica de los programas TACEVAL en los que estamos involucrados, así como en otros muchos temas, incluyendo incluso los docentes donde se constata el hecho de que en un futuro próximo muchas áreas de la enseñanza militar serán desarrolladas en colaboración con otros países de la Alianza.

\section{Introducción}

La reforma que la Alianza Atlántica hizo en 1999 de su Estructura de Mando vino impuesta por el deseo de acomodarse a una situación que preveía, entonces, una disminución del riesgo de amenaza sobre su Territorio, 
así como una mayor probabilidad de tener que actuar fuera del mismo; también en la necesidad de economizar medios y de adaptarse a la situación que planteaba la incorporación de nuevos países miembros como Polonia, Hungría y la República Checa, así como de España que, en aquellas fechas, decidió integrarse de modo pleno en la estructura militar de la misma.

Esta plena integración ha supuesto para el Ejército del Aire, al igual que para el Ejército y la Armada, tener que acometer una serie de cambios para adaptarse a la nueva situación, proceso que todavía es pronto para considerar como definitivamente acabado.

La adaptación ha tenido y tiene diversos aspectos: orgánico, de recursos y de procedimientos.

En el aspecto orgánico, la creación del Centro Combinado de Operaciones Aéreas en Torrejón (CAOC 8) y la transferencia de autoridad de unidades aéreas a la Alianza ha supuesto un cambio importante que ha tenido consecuencias políticas y militares, como es la cesión permanente de unos efectivos (que son la espina dorsal del poder aéreo nacional) así como de determinadas responsabilidades; con la real o aparente pérdida de soberanía nacional que esto conlleva. Todavía hoy, estamos tratando de encajar esta nueva situación que afecta a nuestra propia organización de mando nacional.

En lo que se refiere a recursos materiales, la convivencia de dos centros de operaciones aéreas, uno aliado y otro nacional, así como el uso común de medios aéreos, suponen un reto y un esfuerzo adicional en determinados casos. La inclusión de determinadas unidades en el plan de evaluaciones de la OTAN obligará a la adquisición de materiales de los que se carece y a la modernización de algunos de los existentes.

En lo que respecta al personal, las nuevas exigencias reclaman la dedicación de un importante contingente humano para cubrir las necesidades operativas y de mando. El sistema de evaluaciones de la Alianza ha puesto de manifiesto la necesidad de completar las plantillas de las unidades operativas. También nos encontramos con la nuevå necesidad de los puestos que corresponde cubrir, al Ejército del Aire, en los Estados Mayores de la Alianza e internacionales.

La adecuación, por último, a los procedimientos OTAN lleva consigo un esfuerzo adicional que se traducirá en un cambio en las estructuras educativas, absorbiendo recursos materiales y personales.

Todo esto supone una revolución que afecta profundamente al Ejército del Aire en todos los campos de actuación que le son propios. El cambio no es fácil, porque lo más probable es que deba hacerse, hasta donde se pueda, empleando los recursos existentes, en lugar de contar con otros adicionales. Dada la actual tendencia al crecimiento cero, habrá que ade- 


\section{El Ejército del Aire en la estructura militar de la OTAN}

cuar las estructuras para tratar de adaptarse a las nuevas exigencias, aunque es de temer que esto no sea suficiente y se necesite de una inyección adicional de recursos.

La pregunta es si hay un camino alternativo y si vale la pena el sacrificio que cualquier reforma exige; la respuesta no puede ser más que una, como uno, y único, es el camino: nuestra participación plena en la Alianza es un objetivo político de primer orden y, hoy por hoy, irrenunciable. Por tanto, es mejor ponerse cuanto antes a trabajar para que esa participación sea eficaz y satisfaga por otro lado las aspiraciones y los compromisos que nuestro país tiene respecto a la Alianza, en concordancia, siempre, con los intereses nacionales. El resultado de este proceso de adaptación se ha de traducir, por otro lado, en una modernización y una racionalización eficaz de nuestro Ejército del Aire, adecuando los medios y las estructuras a las nuevas misiones.

Este proceso de adaptación debe ir, naturalmente, de la mano de una revisión estratégica profunda que debe extenderse al resto de los ejércitos, puesto que ellos también se ven afectados por la nueva situación.

\section{Orgánica}

Antes de la incorporación a la estructura militar, el EA contaba con los organismos adecuados, aunque modestos, para sus necesidades nacionales. El Mando de Combate (MACOM) era el mando operativo encargado de planear y ejecutar las operaciones aéreas en ejercicios y, llegado el caso, las reales. Para ello contaba con un Estado Mayor y con un Centro Combinado de Operaciones Aéreas (CAOC), para el planeamiento de detalle y el seguimiento de las operaciones. La doctrina OTAN y las lecciones aprendidas de los conflictos pasados (Irak y Bosnia), así como la experiencia ganada a través de nuestra participación en ejercicios OTAN, se habían incorporado en gran medida a nuestra forma de trabajar, así el uso, por ejemplo, de las ATO (Air Tasking Order) y ACO (Airspace Coordination Order) se venían ya utilizando en todos los ejercicios. Se hacía un uso adecuado de los factores de multiplicación de fuerza como el reabastecimiento en vuelo y los AEW (Air Early Warning), así como de la guerra electrónica. A nivel de conducción táctica se contaba con los COC (Centro de Operaciones Aéreas) de Torrejón y Gando, así como de un sistema de radares de control aéreo.

Con la creación del CAOC-8, dependiente de AIRSOUTH (Mando Aéreo de la Región Sur de la OTAN) se planteó si había que mantener el propio CAOC nacional o si éste debía ser absorbido por aquél. Se decidió que era necesario mantener el nacional para separar las actividades na- 
cionales de las de la OTAN. Sin embargo, en la práctica, esto no es tarea fácil, el motivo es que el espacio aéreo nacional es uno, como también lo son los medios de detección y control transferidos a la OTAN. El uso común de ambos puede dar lugar a conflictos de competencia, por ejemplo con el criterio utilizado para clasificar las trazas aéreas ${ }^{1}$, así como con las normas de actuación (Reglas de Enfrentamiento) que pueden ser diferentes para la OTAN y para la Autoridad Nacional, especialmente si no están al mismo nivel de alerta ${ }^{2}$.

En el fondo, lo que subyace es el hecho de que pertenecer a la Estructura Militar-Defensiva de la Alianza, supone, teóricamente, el que ésta asuma, como colectiva, la responsabilidad de la defensa individual de cada uno de sus miembros, lo cual se traduce en una cesión de soberanía que cuesta comprender y asumir; postura a la que no son ajenos otros países de la Alianza que llevan muchos más años integrados plenamente en la misma.

Esta delegación de responsabilidad no sólo afecta a la defensa aérea del territorio, sino también a la terrestre y naval. La doctrina de la OTAN establece cómo se articula la Estructura de Mando para las operaciones a las que se aplica el Artículo Quinto del Tratado de Washington (defensa del territorio OTAN): El comandante de la FOC (Fuerza Operativa Conjunta) es el CINCSOUTH (Comandante de la Región Sur) y sus mandos componentes subordinados son AIRSOUTH (aéreo) y NAVSOUTH (naval). El mando componente terrestre recaería, en caso de que la amenaza se materializara en la Región Sur-Oeste, en el Comandante del JSRC-SW (Mando Subregional Conjunto del SO- Retamares). Otra variante, para crisis de menor entidad, es que CINCSOUTH delegara el mando conjunto en el Comandante del JSRC-SW, apoyado por los mandos aéreos o navales citados o poniendo fuerzas de éstos bajo el mando del citado JSRC-SW.

En teoría, es posible que AIRSOUTH delegue el mando táctico en el CAOC-8, cuyo comandante, así como el del JSRC-SW es español, lo cual puede dejar nuestra conciencia algo mas «tranquila», aunque esto no es seguro. Por otro lado, ante una posible reducción de CAOCs en la Región Sur, si el CAOC-8 fuera eliminado, no nos quedaría ni siquiera esa posibilidad de mantener cierto carácter "nacional» en la estructura del mando aéreo, responsable directo de la defensa y de las operaciones áreas en nuestro territorio.

Como se ve, las implicaciones de carácter orgánico y doctrinal de nuestra integración son extremadamente importantes y han de exigir un notable esfuerzo intelectual de adaptación para dar respuesta a las incógnitas que se plantean. El quid de la cuestión puede residir en estudiar y analizar cómo los demás países de la Alianza aceptan esta pérdi- 


\section{El Ejército del Aire en la estructura militar de la OTAN}

da de soberanía y cómo concilian los intereses de la Alianza con los puramente nacionales, para seguir una regla de oro: no querer ser más ni menos que otros, dar un margen de confianza a la OTAN, teniendo en cuenta las ventajas que supone el formar parte de una alianza, aún a costa de cierta pérdida de soberanía, y cuidando de mantener siempre aquellos intereses nacionales irrenunciables, que pueden verse afectados por dicha pérdida y que deben ser cuidadosamente vigilados.

Hay que aclarar que, en esta materia, la misma Alianza tiene que clarificar su postura ante las situaciones de crisis. En estas circunstancias, a veces las naciones se adelantan, tomando medidas que la propia Alianza tarda en adoptar. Esto que puede ser causa de conflicto de competencias entre dos sistemas de alerta diferentes, sobre un espacio aéreo común, no deja de ser una discordancia que debe ser analizada y a la que debe ponerse remedio, pues, en caso contrario, pone en entredicho la propia validez de las estructuras de mando y el sistema de trasferencia de competencias, que la Alianza ha establecido en tiempo de paz y que debe ser coherente con los procedimientos a emplear en tiempo de crisis.

\section{Recursos}

La trasferencia del mando operativo de las unidades, o la asignación de las mismas a planes específicos, supone el someterse a la exigencia de disponibilidad y capacidades que la Alianza exige a las unidades que están bajo su mando. El control del estado de las unidades se efectúa por medio del programa de evaluaciones tácticas periódicas (TACEVAL). Los objetivos de estas evaluaciones son:

1. Identificar y asesorar sobre el grado de cumplimiento de las normas OTAN de las unidades.

2. Identificar deficiencias en recursos o capacidades.

3. Recomendar, en su caso, las mejoras a introducir en las capacidades operativas y las desviaciones encontradas respecto a las normas OTAN.

Las unidades a las que se aplica el programa son:

- RF (Fuerzas de Reacción)

- CAOCs y unidades subordinadas:

- Escuadrones asignados a Defensa Aérea

- Unidades de GBAD (Defensa Antiaérea de Bases)

La evaluación se efectúa sobre las áreas operativa y logística, y el resultado puede ser desde «excelente» hasta «insatisfactorio». Respecto a la capacidad para cumplir la misión, se mide desde el grado «superior" hasta el «no operativo». 
La evaluación es llevada a cabo por un equipo de expertos, mediante un ejercicio en el que se juzga la capacidad de la unidad para sobrevivir y operar, la mayoría de los casos, desplegada fuera de la base de residencia habitual, sometida a ataques convencionales y, en su caso, en ambiente NBQ (Nuclear-Biológico-Químico). Lo que se juzga es su capacidad de:

- Mantener el ritmo de operaciones, así como su capacidad de recuperación y de protección.

- Adaptarse a los escenarios

- Adaptarse a los procedimientos OTAN y nacionales

- Adaptarse a situaciones de crisis, contingencia y guerra

- Adaptarse a los SOPs (Procedimientos Operativos Standard)

El año pasado se llevó a cabo la evaluación, a título experimental y por vez primera, de uno de nuestros escuadrones de Fuerzas Aéreas de Reacción, que irá seguida este año de la real correspondiente, estando previsto para el próximo la evaluación de algunos otros. Estas evaluaciones van a poner de manifiesto el estado de nuestras unidades en relación con las normas y capacidades que exige la Alianza.

Uno de los recursos de los que el Ejército del Aire anda escaso es el que se refiere a la dotación de pilotos de las unidades, problema agudizado por la constante sangría de los mismos, que optan por pasar a la aviación civil, mejor remunerada. Este es un problema endémico que otras fuerzas aéreas han resuelto o intentan resolver a través de diversas medidas:

- Aumentando el número de plazas de ingreso en la escala de pilotos.

- Estableciendo un mínimo de tiempo de permanencia en servicio obligatorio para los que reciben instrucción de pilotaje.

- Incentivando la permanencia por periodos de reenganche adicionales, cumplido el tiempo de permanencia obligatorio, mediante primas de reenganche y aumento de sueldo.

- El establecimiento de unidades o individuos de la Reserva o Guardia Nacional, que trabajan a tiempo parcial, especialmente en unidades de transporte, aunque también se da en unidades de caza.

En general, se puede decir que la solución está en una combinación flexible e inteligente de todas las apuntadas, adaptada a las circunstancias de la oferta y la demanda, digamos que con un sentido empresarial realista. Todo un reto para cualquier administración. Sin embargo, no todos son desventajas en esta situación, pues es cierto que la «fuga» de pilotos constituye por si misma una forma de dar salida natural al excedente teórico que se produce en los empleos superiores de oficiales, cir- 


\section{El Ejército del Aire en la estructura militar de la OTAN}

cunstancia que no se da en otros ejércitos, al menos en la magnitud con que se produce en el Ejército del Aire.

No obstante, hay que decir que si bien las medidas apuntadas constituyen herramientas muy útiles para contrarrestar el efecto citado, no debe dejar de considerarse el factor moral. Una de las razones para permanecer o retrasar la incorporación a la vida civil es la satisfacción y el gusto por lo que se hace, el espíritu de cuerpo, el amor al servicio, el sentirse defensor de la Patria y de las causas justas y el saberse protegido y atendido en sus necesidades vitales (personales y familiares), derivadas de una vida dura de servicio, por sus jefes y por la sociedad. Todos estos sentimientos ayudan en gran medida a resolver el problema. Naturalmente dependen de una gran voluntad de todos porque así sea (política de personal) y del empleo de ciertos recursos extras, aunque no tantos si tenemos que recurrir exclusivamente a los ya citados de carácter puramente material.

Relacionado con la dotación de pilotos está el número de horas de vuelo de entrenamiento que cada piloto debe realizar, como mínimo, para estar en el nivel adecuado. Puede que el E.A. no cuente en la actualidad, tal como está estructurado y con arreglo al número de unidades que posee, con los recursos necesarios para generar el volumen de horas de vuelo que se necesitan para alcanzar el nivel requerido. Estos recursos son los repuestos, el personal de mantenimiento y el combustible.

Otro recurso crítico es el personal de apoyo a las operaciones, en las áreas de inteligencia y de preparación de misión, áreas que han sido asumidas siempre por el personal de vuelo, pero que debe separarse convenientemente, puesto que las tripulaciones deben estar empeñadas exclusivamente en el ciclo vuelo-descanso y en los tiempos de preparación y análisis antes y después de la misión.

En el caso de las unidades de Reacción hay que contar con medios que puedan ser desplegables a otras bases, esto incluye material informático, mobiliario de operaciones e inteligencia, comunicaciones para enlazar las áreas de operaciones, mantenimiento, seguridad y GBAD (Defensa Antiaérea). Así como un puesto de mando móvil que, aunque austero, esté dotado de los medios necesarios para el control de las operaciones.

Respecto al mantenimiento, se debe contar con el equipo auxiliar necesario para operar plenamente al ritmo de combate requerido, así como del repuesto necesario para los primeros días. Lo mismo sucede con el armamento y el combustible, en su caso, que puede exigir la necesidad de contar con contenedores de goma circunstanciales.

Atención especial se tendrá que dar a la capacidad de operar en amenaza de ambiente NBQ (Nuclear, Biológico, Química). 
En otro campo, se verá sometido a evaluación el CAOC-8, en el que la nación anfitriona, en este caso España, está absolutamente empeñada. Igualmente sucederá con las unidades subordinadas del Sistema de Alerta y Control.

Está claro que el proceso de evaluaciones ha de ser una herramienta magnífica para poner de manifiesto nuestras deficiencias y así acometer un proceso de mejoramiento de nuestras unidades para adecuarlas a los requisitos de la OTAN. Naturalmente, esto va a suponer el adoptar medidas, algunas de las cuales estarán al alcance del Ejército del Aire; otras, en cambio, dependerán de la aportación de recursos adicionales.

Otro elemento de impacto, ocasionado por nuestra integración plena en la nueva estructura de la Alianza, es el elevado número de personal que ha habido que incorporar a las estructuras de mando: CAOCs, Mandos Específicos como AIRSOUTH, Mandos Conjuntos como SAPHE, SACLANT, AFSOUTH y el JSRC-SW de Madrid. En total supone una aportación de 74 nuevos oficiales, en su mayoría postgraduados con el curso de Estado Mayor. Este número equivale prácticamente a tres promociones enteras, descontadas las bajas que históricamente, por diversos causas, se vienen produciendo en cada promoción. De momento ha habido que recurrir al personal existente en la actualidad, muy mermado en número por las razones antes apuntadas, lo cual ha supuesto el dejar en cuadro la propia estructura de mando del E.A, afectando, también, a nuestra obligada aportación de personal al resto del Ministerio de Defensa. Es evidente que para compensar esta nueva exigencia de personal habrá que aumentar el número de integrantes de las promociones futuras, amén de los ahorros que se puedan conseguir a través de una reestructuración o reducción adecuada.

\section{Procedimientos}

Si importante es el impacto que la nueva situación tiene sobre los actuales recursos disponibles, no lo es menos el que tiene sobre la forma de trabajar del personal y del propio E.A., como institución.

Los nuevos parámetros a los que tienen que acomodarse las unidades van a suponer un nuevo enfoque en las formas de entrenamiento de las mismas, que habrá de orientarse plenamente a las nuevas necesidades operativas. Algunos criterios que se daban por buenos tendrán que ser revisados y otros adaptados a las nuevas circunstancias.

Hasta que ocurrieron los recientes ataques terroristas contra el corazón político, militar y económico de los EE.UU., se consideraba como 


\section{El Ejército del Aire en la estructura militar de la OTAN}

muy poco probable el que, en un futuro próximo, la Alianza se viera amenazada en su propio territorio. Por ello, se enfatizaba la necesidad de mejorar la capacidad de despliegue y respuesta de la Fuerzas Armadas por cuanto los posibles escenarios se iban a dar fuera, cerca o lejos, del territorio nacional de los países miembros. Todavía, es presumible que esto pueda suceder, incluso para ejercer una defensa a distancia contra la amenaza terrorista, por lo que continua vigente la cultura expedicionaria, en la que algunos países de la Alianza disfrutan de una mayor experiencia.

La aviación de transporte militar es uno de los componentes mas importantes para poder desplegar la fuerza y es evidente que Europa, en concreto, está haciendo un gran esfuerzo para no depender tanto de los EE.UU. en este terreno. No obstante, no es sólo la capacidad de transporte lo que cuenta. Las propias unidades de combate necesitan una notable transformación en sus estructuras de personal y material para hacerlas expedicionarias, así como de cambio de mentalidad que se basa en la elaboración de nuevos procedimientos y el entrenamiento conveniente para familiarizarse con este nuevo entorno.

Es evidente que el despliegue de nuestras unidades en el Teatro de Operaciones de los Balcanes constituyó un avance y una forma de aprender los nuevos modos, sobre todo cuando se efectuó el despliegue inicial. También la participación en los ejercicios tipo RED-FLAG ${ }^{3}$ han aportado magnificas enseñanzas al respecto.

Respecto al personal que está integrado en los Estados Mayores de la OTAN, el encuentro con unas formas distintas de trabajar, con procedimientos diferentes a los se estaba acostumbrado en nuestras Fuerzas Armadas, ha supuesto un choque tremendo, que en muchos aspectos se ha traducido en un gran esfuerzo para el mismo. Si a ello se añade la dificultad de tener que trabajar en una lengua (inglesa) que no es la materna, puede imaginarse lo que ha supuesto para la primera tanda de personal que se han incorporado a los Estados Mayores de la Alianza.

La colección de reglamentos y normas que rigen el funcionamiento de los Estados Mayores de la OTAN es numerosa y no era desconocida para el E.A, pero una cosa es saber que está ahí y otra es trabajar con ella. La burocracia OTAN no es ni más ni menos complicada que la de nuestras Fuerzas Armadas, simplemente es distinta.

La descripción que a continuación se hace de esa burocracia OTAN está basada en la experiencia propia que se ha obtenido en el Estado Mayor de AFSOUTH, pero es extensible, por similar, a cualquier otro E.M específico, incluido el aéreo.

Es distinto por ejemplo el proceso de la decisión. Nuestro sistema es más jerárquico, si una Sección del E.M de un órgano subordinado elabo- 
ra un informe, éste, antes de ser remitido a un organismo superior, debe pasar por toda la cadena de mando para su aprobación. Por lo general, la Sección elabora su informe de modo independiente, a lo sumo en consulta con otros organismos de su mismo nivel o sobre la base de otros informes inferiores. En la OTAN es normal que el oficial que hace el informe se cartee por correo electrónico con su homólogo del organismo superior, para contrastar sus opiniones sobre el asunto. En general se puede decir que se coordina mucho al nivel de «staff», gracias al uso extensivo del correo electrónico. Esto, en principio, es muy útil por cuanto agiliza el proceso de la decisión, como se ha comprobado recientemente en la distintas operaciones OTAN, que la situación crítica de Macedonia ha obligado a poner en marcha, como la Operación Harvest y la Amber Fox.

El planeamiento en los cuatro niveles, Político (Consejo Aliado), Estratégico (SHAPE), Operacional (AFSOUTH) y Táctico (Task Force Harvest y Amber Fox), se ha efectuado de modo paralelo y simultáneo, de modo que cuando se ha tomado la decisión al más alto nivel, prácticamente los planes de operaciones en los restantes niveles estaban ya concluidos. Lo que por un sistema piramidal, de abajo arriba y viceversa, hubiera costado semanas, pudo hacerse en días.

Sin embargo, este sistema no está exento de riesgos: si la cadena de mando (sistema piramidal) no está perfectamente informada del trabajo que se realiza en la periferia (sistema matricial o lateral), existe el peligro de incurrir en una cierta forma de indisciplina administrativa que causa no pocos problemas, dando lugar a una promiscuidad indeseable en la que la cadena de mando puede verse, en cierto modo, marginada del proceso.

No obstante, en los asuntos relevantes, la decisión final de aprobación se lleva a cabo por el procedimiento ordinario en el que el trabajo pasa en papel escrito (paquete), acompañado de toda la información necesaria, de la División de Estado Mayor a la oficina del DOS (Director del Staff), que actúa como una especie de Secretaria de Estado Mayor, y que lo hace pasar por todo el Grupo de Mando, de abajo arriba (Segundo Jefe del Estado Mayor, Jefe del Estado Mayor, Segundo Comandante y Comandante). $\mathrm{Al}$ final, el documento, una vez aprobado y firmado por el Comandante, es anticipado al destinatario por correo electrónico, aunque también se remite por correo ordinario.

Una de las virtudes de este correo electrónico es que permite la utilización del mismo hasta el nivel de Secreto por lo que se puede emplear con la mayoría de la documentación que se maneja.

Las directrices de trabajo que se dan al Estado Mayor se proporcionan mediante la «Tasker» (Tarea), emitida por el Comandante o por cual- 
quiera de los componentes del Grupo de Mando, siempre a través del DOS, que se encarga de canalizar la tarea a las Divisiones o a los Comandantes Subordinados, siendo el responsable de fijar y controlar los tiempos de ejecución de las misma.

Las tareas se encargan a una División o a varias, asignándose a una concreta como responsable de efectuar la coordinación, que se realiza mediante consultas, reuniones o establecimiento de grupos de trabajo.

Un organismo importante en un Estado Mayor OTAN es el JOC (Joint Operation Center), éste suele depender de la División de Operaciones y es el encargado de seguir los acontecimientos operativos y tener informado de los mismos al Grupo de Mando. Actúa en coordinación con las Divisiones y está enlazado con los respectivos JOCs del Mando Superior y de los Mandos Subordinados. En caso de operaciones reales como las de los Balcanes, los JOCs están activados las 24 horas del día. El proceso de información cruzada se lleva cabo mediante informes periódicos y comunicaciones puntuales.

Otro aspecto a destacar es el uso extensivo del trabajo en equipo, mediante la celebración de reuniones periódicas u ocasionales. Así el Grupo de Mando se reúne todos los días a primeras horas de la mañana, para recibir el informe del Director del JOC y del Jefe de la División de Operaciones sobre la situación en el Teatro. Después se tratan los asuntos del Cuartel General y de la Región.

El Jefe del Estado Mayor suele tener una reunión con los Jefes de División, al menos una vez por semana.

Asimismo, semanalmente, se tiene una reunión con los Mandos Subordinados para tratar de la situación en los Balcanes y otra, también, en la que se tratan los asuntos de tipo general. En ocasiones, se hace uso de la video-conferencia para relacionarse con aquellos mandos que no están asentados en la proximidad del C.G.

Además existen reuniones de más alto nivel que se celebran, varias veces al año, como:

- RCC (Conferencia Regional de Comandantes)

- AFNORTH- AFSOUTH (conferencia entre los dos comandantes y sus E.Ms)

- SOUTHERN VISION (CINCSOUTH con todos los Mandos Subordinados de la Región Sur)

- COS-CONFERENCE (lo mismo que la anterior pero a nivel de COS -Jefes de E.M).

Aparte de estas conferencias es normal que las Divisiones celebren otras, a su nivel, con los homólogos de los Mandos Subordinados. Esto sin contar con un sinfín de reuniones que son convocadas por SHAPE, en dis- 
tintas áreas y que en su mayoría están instituidas como convocatorias periódicas.

Notable es el grado de perfeccionamiento del proceso de planeamiento de carácter operativo. Los pasos y el tipo de documentos a utilizar para la confección de directivas y ordenes de operaciones, están muy definidos y constituye en sí mismo un método excelente de estructurar de un modo eficaz el proceso de la decisión, desde el nivel estratégico al táctico, pasando por el operacional.

Sorprende el grado de ayuda que tiene el Comandante en todas las tareas del desempeño del mando. No hay asunto que no esté perfectamente documentado. Así, los paquetes mencionados anteriormente van precedidos de un «Point Paper» que resume el contenido de los mismos y en el que firman, con su aprobación o no, las Divisiones que han participado o han sido consultadas sobre el asunto. Al trabajo se acompaña toda clase de documentos de referencia. En la carátula de la carpeta suelen firmar todos los componentes del Grupo de Mando, con sus observaciones, sobre la misma carátula o en informe separado que se incluye a la carpeta.

En otro orden de cosas, sabido es el amplio programa de ejercicios, de todo tipo, que la Alianza realiza periódicamente. Estos pueden ser de Puesto de Mando (CPX) o con participación de fuerzas reales (LIVEX), similares a los que se realizan en el ámbito nacional. Sin embargo, hay un tipo de ejercicios que es peculiar, no tan común, que es el de preparación de Cuarteles Generales que han de hacerse cargo de una misión concreta, este es el caso de los últimos Cuarteles Generales de KFOR en Kosovo, que se relevan cada seis meses y que han pasado por lo que podríamos llamar la «Universidad de los Balcanes», aquí en AFSOUTH.

Estas actividades, que se consideran más como una forma de instrucción que como un ejercicio, abarcan desde conferencias por expertos, hasta simulaciones en las que se trata de presentar casos parecidos a los que el futuro Cuartel General se va enfrentar en la vida real: se crean situaciones ficticias de crisis a las que hay que hacer frente, reuniéndose el Cuartel General en gabinete de crisis con la participación de todos los jefes de área que sean necesarios. Dado el carácter complejo de las misiones que desempeñan cualquiera de los dos Cuarteles Generales (SFOR y KFOR) que la OTAN tiene en los Balcanes y sus posibles variables, que van de lo estratégico a lo táctico, pasando por lo político, humanitario, orden público, publicitario, etc., son múltiples los escenarios ficticios los que pueden componer la escenificación.

En definitiva, se trata de un entorno nuevo con el que se ha encontrado el primer grupo de personal que ha accedido a la estructura de estos 


\section{El Ejército del Aire en la estructura militar de la OTAN}

Cuarteles Generales. Es evidente que, éste y aquél otro que le está sustituyendo, ha tenido la inmensa fortuna de ampliar sus conocimientos profesionales en una organización militar que goza de una solera y un rigor dignos de tenerse en cuenta. Si el impacto para ese personal ha sido significativo, igualmente lo va ser para el Ejército del Aire su vuelta de aquellos destinos. La experiencia que arrastra va a influir en los modos y comportamientos internos, por cuanto es inevitable el efectuar comparaciones y el espíritu crítico, siempre saludable, va a desembocar en propuestas y modificaciones para acercar nuestra forma de actuar a la de la Alianza.

Aunque no todo sea imitable, porque no lo es, es evidente que se encontrarán campos en los que la innovación será beneficiosa. En cualquier caso, será ventajoso el que nuestros procedimientos se asemejen lo más posible a los de la Alianza, ello facilitará la coordinación entre el Ejército del Aire y aquélla. Por otro lado, facilitará tremendamente la incorporación de nuestro personal a puestos de la OTAN y viceversa.

No será, por otro lado, tarea fácil. Las estructuras son resistentes al cambio por naturaleza y las nuevas ideas, que ese personal recién incorporado a su destino ha de aportar, puede, en un principio, que no sean aceptadas o valoradas en su medida. Sin embargo, en un futuro muy próximo, no habrá diferencias de criterio ni suspicacias porque todo el personal de Estado Mayor, tarde o temprano, habrá tenido la oportunidad de pasar por la OTAN; experiencia que se considerará como imprescindible para la formación de cualquier oficial de estado mayor.

Debería pensarse la forma de evitar el choque que ha supuesto, para la primera remesa de personal incorporado a puestos de la OTAN, el encontrarse con una situación nueva y para la que no ha existido una preparación específica. Se debería aprovechar la experiencia que ya se posee, para entrenar a los que fueran a incorporarse a los citados puestos. El sistema educativo debiera adaptarse a esta nueva exigencia. La formación debe ir dirigida a dos grupos de personal diferentes:

- El que se incorpora a unidades y Cuarteles Generales de los Balcanes y otros organismos o unidades empeñados en misiones de paz. La preparación previa a la incorporación es aquí muy importante, piénsese que normalmente a estos destinos se va por un tiempo limitado de, normalmente, seis meses. Al individuo se le necesita rindiendo en su trabajo desde el primer día, por lo que debe conocer perfectamente la naturaleza de su trabajo y además el entorno en que va a desempeñarlo. No hay tiempo para un entrenamiento «in situ», se pide que empiece a rendir desde el primer día. El conocimiento de una lengua extranjera, si se exige, debe ser el adecuado para poder desempeñar su cometido. 
- El que va a ocupar puestos en Cuarteles Generales o unidades multinacionales por un periodo largo de tiempo. La preparación previa no es tan crítica, pero es más que deseable. El idioma tiene asimismo gran importancia.

Es notable el grado de especialización y competencia que se observa en otros ejércitos que pertenecen a la Alianza. En general, se puede decir que es difícil ver al oficial que sirve para todo y la tendencia es que, dentro del Ejército, Arma o Especialidad Fundamental, el profesional desarrolla sus cometidos a través de toda su vida militar en una especialidad concreta. La especialización llega hasta niveles muy particulares, algunos poco conocidos para nosotros, como especialistas en planeamiento, guerra psicológica y prensa, por poner algunos ejemplos.

\section{Conclusiones}

Después de la integración de España en la estructura militar de la OTAN, se puede decir que las Fuerzas Armadas han experimentado y están experimentando un cambio importante. Un acontecimiento que delimita un antes y un después. Esto afecta igualmente al Ejército del Aire, como parte de esas Fuerzas Armadas.

Los cambios abarcan aspectos relacionados con la organización, el material, el personal y los procedimientos. Algunos de éstos obligan a un cambio de mentalidad que debe formalizarse a través de la enseñanza y el entrenamiento, para adaptarse a los nuevos modos de actuación que exige la pertenencia a una organización tan rodada como la Alianza. En la medida en que nuestra forma de actuar y el entrenamiento se adapte a los estándares OTAN, más fácil será, para nuestro personal, el pasar de un destino nacional a otro OTAN y viceversa. Es deseable, desde todos los puntos de vista, que nuestros procedimientos sean los más parecidos a los de la OTAN. Este es un esfuerzo que requiere de un sistema educativo que se adapte a las nuevas exigencias y debe extenderse a las escuelas específicas del Ejército del Aire y a la Escuela Superior de las Fuerzas Armadas.

Por otro lado, la exigencia de calidad y especialización supone que se dediquen recursos a esa enseñanza. Los recursos no son tanto cuantitativos como de orientación. Hay áreas en que necesitamos una mayor profundidad en las materias y en la extensión de los conocimientos. Un campo, en el que la misma OTAN es deficitaria porque no le llegan los recursos humanos pedidos, es el de las tecnologías de la información. El E.A, que abandonó el impartir cursos DIN (Diplomado en Informática) confiando en que la Escala Media proporcionaría los recursos necesarios, 


\section{El Ejército del Aire en la estructura militar de la OTAN}

debe estudiar el modo de paliar la actual carencia de personal en una área que es vital para el desarrollo tecnológico.

Para satisfacer las necesidades educativas en determinadas áreas, que son comunes a las de los otros Ejércitos, sería deseable el hacer un esfuerzo conjunto para la creación de los centros de enseñanza o la programación de los cursos adecuados, incluso recurriendo a los centros educativos civiles, por cuanto a la larga, si satisfacen los requisitos, han de resultar más económicos.

Para un ejército pequeño, como el nuestro, el dedicar recursos humanos a la enseñanza supone un coste muy elevado que va en detrimento de la tarea fundamental operativa. Una revisión valiente de nuestras estructuras educativas llevaría a la conclusión de que muchas de las actividades docentes que ahora son desempeñadas por nuestro personal podrían serlo por entidades externas al E.A.

Obsérvese, por ejemplo, como Alemania forma a sus pilotos enteramente fuera del país: desde la enseñanza de pilotaje elemental, a cargo de Lufthansa, hasta la básica y avanzada, en EE.UU, a través de un programa conjunto en el que intervienen varias naciones, con lo cual, además, después de casi dos años en EE.UU, se asegura para los alumnos un buen conocimiento del idioma inglés, tan necesario en aviación como en la OTAN.

En el área de recursos tanto humanos como materiales, la exigencia de autenticidad que la OTAN impone y controla por medio de su programa de evaluaciones periódicas, así como la especialización y competencia que son necesarias, para que nuestras FF.AAs puedan actuar junto a otras fuerzas de la Alianza, requiere de un planteamiento de revisión con profundidad.

Es necesario dotar a nuestras unidades aéreas del personal en número y calidad suficiente, por lo que habrá que pensar en suprimir aquello que no contribuya de un modo sustancial a la misión. Dado que el techo de personal es difícil que se eleve, para aumentar la plantilla operativa habrá que reducir el número de personal que se dedica a otras tareas. Esto supone ajustar el número de Estados Mayores y organismos al mínimo imprescindible, evitando redundancias y orientándolos a la ejecución o apoyo a las tareas operativas.

Los Estados Mayores que queden deberán asumir y reunir en sí mismos un mayor número de funciones y tareas. Mención especial, no obstante, se debe hacer de la sustanciosa demanda que la OTAN hace de nuestros oficiales de E.M., necesidad adicional, de estos últimos tiempos, que camina en dirección contraria a esa corriente de ahorro mencionada y que exige de un aporte de recursos extraordinarios. 
Respecto a los recursos materiales, esa misma exigencia parece aconsejar una adaptación del objetivo de fuerza a los recursos económicos disponibles para un sostenimiento adecuado de las unidades. De nada vale el mantener unidades que no se pueden sostener adecuadamente y que por tanto no van a pasar la homologación. Habrá que pensar, probablemente, en la reducción de unidades, al número adecuado que permita un sostenimiento integral de las mismas. Por otro lado, el coste de inversión de capital asociado a unidades que no se emplean a pleno rendimiento supone un lastre demasiado pesado y que incide sobre la disponibilidad de recursos que deben emplearse en nuevas inversiones, ya sea por la necesidad de sustituir el material obsoleto o por la de mejorar el existente que, dado el constante avance tecnológico, debe ser mantenido en un estado de eficiencia razonable.

Esta política de reducción, de tener menos pero mejor, ha de tener impacto sobre las bases desde la que operan las unidades. A menos unidades, menos bases y mejor aprovechadas. Es claro que una concentración de unidades por tipo de material ha de redundar en ahorro de determinados elementos, como talleres de mantenimiento, bancos de prueba y recursos humanos asociados al sostenimiento de la fuerza. No obstante, hay un factor táctico que siempre debe tenerse en cuenta y es que una excesiva concentración de medios redunda en una mayor vulnerabilidad. También pueden existir condicionantes tácticos que obliguen el mantener un despliegue que, aunque no sea rentable económicamente, ofrezca, sin embargo, ventajas tácticas apreciables. Sin mencionar algunos aspectos político- estratégicos que pueden aconsejar a mantener ese despliegue determinado; no debe olvidarse el factor disuasorio que la sola presencia de unidades militares representa y que no puede ser cuantificado económicamente

Es claro que una evaluación de las amenazas debe estar siempre presente a la hora de efectuar una reestructuración dictada por factores económicos. En el caso de un país cuya estrategia estuviera basada en su capacidad de proyección, los factores económicos privarían por encima de cualquier consideración a la hora de diseñar un despliegue estático, pero este caso es mas bien raro por cuanto, en mayor o menor medida, todas las naciones pueden verse amenazadas en su territorio, hasta las que se consideraban absolutamente invulnerables, y tenemos un ejemplo trágico pero bien significativo, en los recientes ataques terroristas al corazón del poder político, económico y militar de los EE.UU de América.

Nuestra geografía peninsular, insular y marítima exige que esos factores sean tenidos en cuenta, aunque sólo sea para mantener una pre- 


\section{El Ejército del Aire en la estructura militar de la OTAN}

sencia en un área de indudable valor estratégico, para nosotros, para Europa y para la Alianza Atlántica.

¿Cambio, reforma?: está claro que los tiempos y los recursos disponibles así lo exigen, pero estos cambios no pueden realizarse de modo aislado. Aunque quisiera, el E.A no puede acometer una reforma en profundidad de sus estructuras y de sus medios de actuación, si no es en el marco de una estrategia conjunta, que debe definir con precisión cual es el objetivo de fuerza conjunto, posible y necesario, para hacer frente a las amenazas y a los compromisos nacionales y colectivos, en el marco de una política de defensa bien articulada.

La entrada de España en la Estructura Militar de la Alianza, así como los últimos acontecimientos, el ataque terrorista a EE.UU, que involucran a la OTAN en un conflicto sin precedentes, hasta el punto de verse obligada a aplicar el Artículo Quinto del Tratado de Washington, imponen un nuevo reto a nuestras Fuerzas Armadas. Estas tendrán que reestructurarse para hacerse más eficaces y más preparadas para las nuevas contingencias a las que hacer frente. Si embargo, no todo puede hacerse a través del ahorro y la reducción, también esto tiene un límite por debajo del cual no se puede llegar sin pérdida sustancial de las capacidades que se nos exigen. Como dice el Secretario General de la OTAN «el esfuerzo extra para combatir el terrorismo requiere de más dinero...Para la OTAN, el síndrome «crecimiento cero", que muchos aplican a la seguridad y defensa es una respuesta insuficiente, y quizás irresponsable, a las nuevas necesidades de seguridad del siglo XXI ${ }^{4}$.

Tener unas metas claras no es tarea fácil para ninguna sociedad humana, encontrar el modo de como conseguirlas es aún más difícil; disponer de los recursos adecuados para llevar a cabo el proyecto, casi imposible; pero si hay voluntad y se está convencido de lo que se persigue, hay posibilidad de éxito; es cuestión de ponerse a ello.

\section{Notas}

1 Traza aérea. Contacto radar de un vehículo aéreo detectado por el Sistema de Control Aéreo.

2 Alerta Aérea. Existen diversos niveles de alerta, según el grado de la amenaza, que se traducen en distintos niveles de disponibilidad de las fuerzas aéreas de respuesta inmediata, así como en diferentes reglas de enfrentamiento.

${ }^{3}$ RED FLAG es un tipo de ejercicio que se realiza en los EE.UU y en el que vienen participando regularmente las unidades del Ejército del Aire.

5 Conferencia de Seguridad de Berlín de 1-9-01. 